\title{
Situational Factors of Influencing Drivers to Give Precedence to Jaywalking Pedestrians at Signalized Crosswalk
}

\author{
Xiaobei JIANG ${ }^{1,2}$ \\ ${ }^{1}$ Department of Transportation Engineering, Beijing Institute of Technology, Beijing 100081, China \\ ${ }^{2}$ Lehrstuhl für Ergonomie, Technische Universität München, München 85747, Germany \\ Wuhong WANG*, Yan MAO \\ Department of Transportation Engineering, Beijing Institute of Technology, Beijing 100081, China \\ Klaus BENGLER, Heiner BUBB \\ Lehrstuhl für Ergonomie, Technische Universität München, München 85747, Germany \\ Received 13 July 2011 \\ Accepted 25 November 2011
}

\begin{abstract}
A large number of fatalities are caused by the vehicle-pedestrian accidents. Under a potential conflict between the vehicle and jaywalking pedestrian, giving precedence to the pedestrian will be a proper decision taken by the driver to avoid collision. Field traffic data has been collected by video recording and image processing at two signalized crosswalks. Vehicle speed performance in the single vehicle-pedestrian encounter and platoon vehicle-pedestrian encounter were analyzed for understanding the driver behavior in the conflict process. Binary logit model was proposed to estimate the drivers' giving precedence influenced by the situational factors and the model was validated to predict the drivers' choices accurately. The vehicle speed, pedestrian speed, pedestrian lateral distance and the vehicle longitudinal distance to the conflict point were proved to affect the drivers' choices in platoon driving. The research results would hopefully be helpful to the design of intelligent vehicles and pedestrian protection systems by the knowledge-based decision making process.
\end{abstract}

Key words: driver behavior; binary logit model; knowledge-based decision making; jaywalking pedestrian

\section{Introduction}

Motor vehicle-pedestrian accidents are a serious problem throughout the world ${ }^{20}$. Pedestrian deaths alone cover about 11 percent of all motor vehicle deaths in the US $^{1}$ (2003), and about 14\% in Germany ${ }^{2}$ (2006-2009). In China, pedestrians make up of more than 20 percent of the traffic accident fatalities (MPSPRC Report ${ }^{3}$ :
$24.6 \%$ in 2003, 24.97\% in 2004 and $25.85 \%$ in 2007), while most of that had resulted from the conflicts with motor vehicles. Considered the factors causing traffic accidents, the driver error accounts for $70 \% \sim 80 \%$ and the pedestrian traffic violation accounts for $15 \%{ }^{3}$ The statistic shows that the proportion of pedestrian fatality in the urban area (67.93\%) is significantly higher than that in the suburban area and rural area ${ }^{4}$. So how can we imagine such a situation that a driver conducts an

\footnotetext{
*Corresponding author: wuhwang@263.net
} 
incorrect maneuver in response to a jaywalking pedestrian at urban crosswalk? Studies on the vehicle driver behavior and the factors of influencing the behavior with regard to giving precedence to pedestrian may provide information for the solution of pedestrian protection systems and promote the motor vehicle active safety $^{23,24}$.

While most of the traffic accidents to a certain extent depend on vehicle speed, vehicle speed also plays an important role on the description of pedestrian safety problems. Many studies on the motor vehicle-pedestrian conflict were centered drivers' speed performance and its effect on yielding safety. Pasanen ${ }^{5}$ indicated at a collision speed of $50 \mathrm{~km} / \mathrm{h}$ the risk of fatal injury for a pedestrian is almost eight times higher compared to a speed of $30 \mathrm{~km} / \mathrm{h}$. High vehicle speed was proved to influence the pedestrian safety greatly according to the empirical evidence $^{6}$ and field observation ${ }^{7}$. Further, the relationship between speed level and pedestrian death risk was analyzed ${ }^{8}$. Using a generalized ordered probit model, the research examines the impact of personal and environmental characteristics on severity of injuries sustained in pedestrian-vehicle crashes, the results suggest that the environmental conditions should be an important consideration when evaluating and planning for pedestrian safety ${ }^{21}$. The evaluation of crosswalk warning system was developed to increase the rate of giving way to pedestrians ${ }^{22}$. In addition, a microscopic traffic simulation model ${ }^{9}$ was proposed to examine the vehicle speed characteristics of interactions with pedestrians, cyclists and other road users. How the speed behavior influenced to give the precedence was investigated by the yielding frequency under different encounter situations related to pedestrian presence ${ }^{10}$ and speed limit ${ }^{8}$.

Although speed can be considered as an important parameter in yielding behavior, it is not the only factor that directly determines the driver's giving precedence to pedestrian because within the vehicle-pedestrian conflict process, other situational information would be interpreted by the driver from the environment. Some researches gave different considerations to describe the driver behavior in giving precedence. Persson ${ }^{11}$ reviewed on communication between road users, and found that the likelihood of a driver giving precedence increased if information of the pedestrian's intention was increased by way of combination of various forms of signs. Many other factors ${ }^{12-14}$ were presented to have an influence to the drivers to give way to crossing pedestrians: the type of crossing and lane, distance between oncoming vehicle and pedestrian, orientation of pedestrian, number of pedestrians, pedestrian distance from the curb, etc. Logit models ${ }^{13,15,16}$ were employed to analyze and evaluate the behavior of pedestrians and vehicle drivers at crosswalks, and it would be the first step for modeling the vehicle-pedestrian encounter process as well as the accident prediction.

However, in the developing countries, the drivers always scramble for the limited space with pedestrians and deter them from having the right of way. At signal controlled crosswalk (with both vehicle signal and pedestrian signal), the drivers may take it for granted that they just follow the traffic signal, focus on the vehicles in front and reduce the detection to the jaywalking pedestrian. As a result, the driver may not make an appropriate decision of giving priority to the jaywalking pedestrians, at the same time the pedestrians will easily be caught in an extremely dangerous situation which was investigated in this study and should be highlighted in the further vehicle-pedestrian conflict analysis.

\section{Methodologies and Data Collection}

\subsection{Field observation}

Two observation crosswalks without any roadside parking are selected for this study. The first one is a minor arterial crosswalk at a signalized $\mathrm{X}$-intersection in Changchun city center. The conflicts between the straight-going vehicles entering the intersection and the jaywalking pedestrians are observed. The average flow rate of the straight-going vehicles (for each observation lane) is $297 \mathrm{veh} / \mathrm{h} / \mathrm{ln}$ within which the passenger cars are about $91.4 \%$ and the average pedestrian flow rate (for the observation crosswalk) is $123 \mathrm{ped} / \mathrm{h}$ with $13.8 \%$ of jaywalking pedestrians. The second crosswalk is a mid-block crosswalk on a two-way undivided urban minor arterial road in Beijing. The average flow rate for each observation lane is $324 \mathrm{veh} / \mathrm{h} / \mathrm{ln}$ with $94.2 \%$ of passenger cars and the average pedestrian flow rate is $107 \mathrm{ped} / \mathrm{h}$ with $26.2 \%$ of jaywalking pedestrians. The crossing markings were clearly visible in these crosswalks. 
Two vehicle-pedestrian encounter situations are defined in this study:

- Situation I: A single vehicle encounters a jaywalking pedestrian;

- Situation II: Vehicle in platoon encounters a jaywalking pedestrian.

Considered the observed zebra crosswalks are perpendicular to the road lane and the evasive driving of the subject vehicle could be treated as a certain lane-based movement, the encounter situation is a right-angle encounter. Passenger cars both in single-driving and platoon-driving status are defined as subject vehicles in the study. Pedestrians who use the zebra crosswalk or very close to the zebra (less than $2 \mathrm{~m}$ ) to cross the road during the pedestrian red time are observed as sample jaywalking pedestrians. Some pedestrians who attempt to violate the signal and head toward the road but are forced to wait on the crosswalk could also be counted.

The driver would theoretically need to notice the mid-block crosswalk $38 \mathrm{~m}$ before reaching it at a speed of $11 \mathrm{~m} / \mathrm{s}$, and have a clear view of both sides of crosswalk from that distance to effectively scan for pedestrians ${ }^{17}$. Considered the average speed of the vehicle at the two study sites is lower than $11 \mathrm{~m} / \mathrm{s}$, a $35 \mathrm{~m}$ lane length before the crosswalk is chosen for this study to estimate the drivers' giving precedence to pedestrian. The observation time is three successive hours on working day in the interval 14:00-22:00 , it is just because the traffic fatality rate is higher than the others according to the Annual Report of the China Road Traffic Accidents Statistics ${ }^{3}$.

\subsection{Video recording of driver and pedestrian behavior}

Many available experiments have been conducted to get the real traffic data, and among the various methods for data collection, video observation is undoubtedly a cost-efficient one to reproduce the traffic event. In this study, the video recording and image processing are introduced as the major data collection methods for the analysis on the factors influencing drivers to give way to jaywalking pedestrians. Fig.1 is a sketch map taking an intersection in Beijing for instance and shows the location of the observation point with the camera view covering the entire length of zebra crosswalk and a 35m approaching lane before the crosswalk.

The movement of the subject vehicle can reflect the driver's approaching behavior to an actual encounter situation. And this proximity can be defined by the distance in space or the distance in time between two road users. Fig. 2 illustrates the timeline of the yielding process by a vehicle B approaching a pedestrian A with the space to conflict point as its y-axis. From studying the trajectories of the subject vehicle, the jaywalking pedestrian and the kinematic equations of motion in relation to a fixed coordinate system, basic characteristics that describing the individual behavior can be derived.

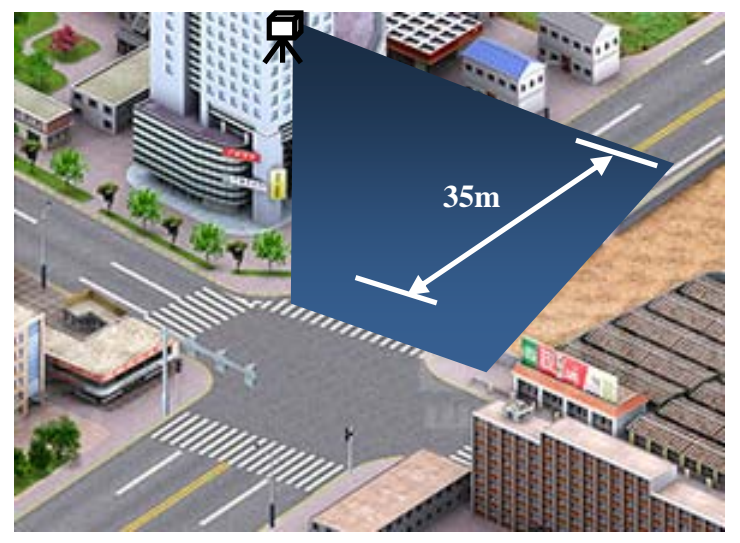

Fig.1 Location of the camera and the observation view

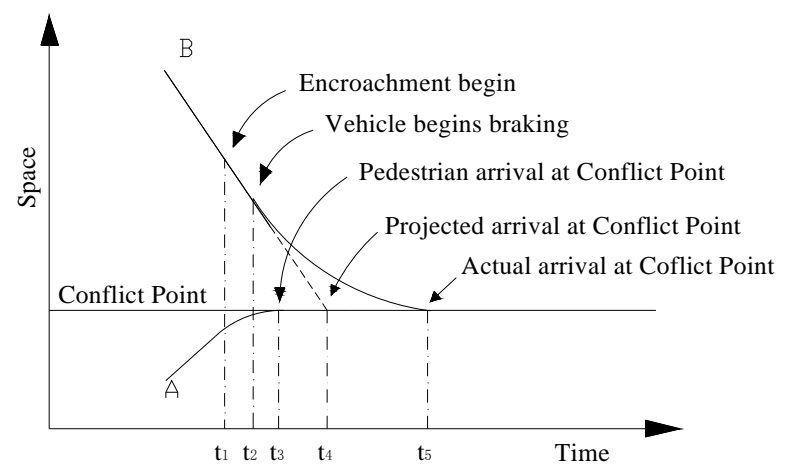

Fig.2 The yielding situation in a conflict

The global polynomial model was applied to transform the image coordinates to road coordinates with the bellow general equation: 


$$
\begin{aligned}
& x=q(X, Y)=\sum_{t=0}^{n} \sum_{j=0}^{n} a_{t j} X^{t} Y^{j} \\
& y=q(X, Y)=\sum_{t=0}^{n} \sum_{t=\varepsilon}^{n} b_{t j} X^{t} Y^{j}
\end{aligned}
$$

Where, the $(x, y)$ denotes the road coordinate and $(X, Y)$ denotes the image coordinate. The value $n$ will influence the accuracy of global polynomial method in the $n$-th power function. Generally, when $n$ equals to 2, nine control points with their image coordinates and road coordinates were measured and substituted into the Eq. (1) and (2) for calculating the transformation coefficients $a_{i j}$ and $b_{i j}$.

\section{Data Analysis}

\subsection{Single vehicle encounter with jaywalking pedestrian}

In naturalistic traffic environment, when a motorist encounters a pedestrian, generally the decision made by a driver is decelerating before the conflict point to avoid a collision. The driver prefers to slow down rather than stop unless s/he is caught in a serious situation. This conflict event can be described as "pursuit - encounter - deceleration - undisturbed passage”. In the first observation site, 52 samples of drivers' giving precedence to jaywalking pedestrians in Situation I are measured based on the trajectory data series of the encounter participants.

The approaching speed is one of the essential parts to describe the vehicle movement in the yielding process and it is also a basic parameter for the measures rating conflict severity. The analysis on the situational factors influencing approaching speed can reflect the driver's maneuver to a certain traffic condition. Fig.3 plots the vehicle speed performance related to its longitudinal distance to the + . It describes a downward trend in vehicle speed with the decrease of the longitudinal distance to conflict point, and vehicle drivers brake from about $10 \mathrm{~m} / \mathrm{s}$ to a relatively low speed at about $4 \mathrm{~m} / \mathrm{s}$ before the conflict point then finally give precedence to

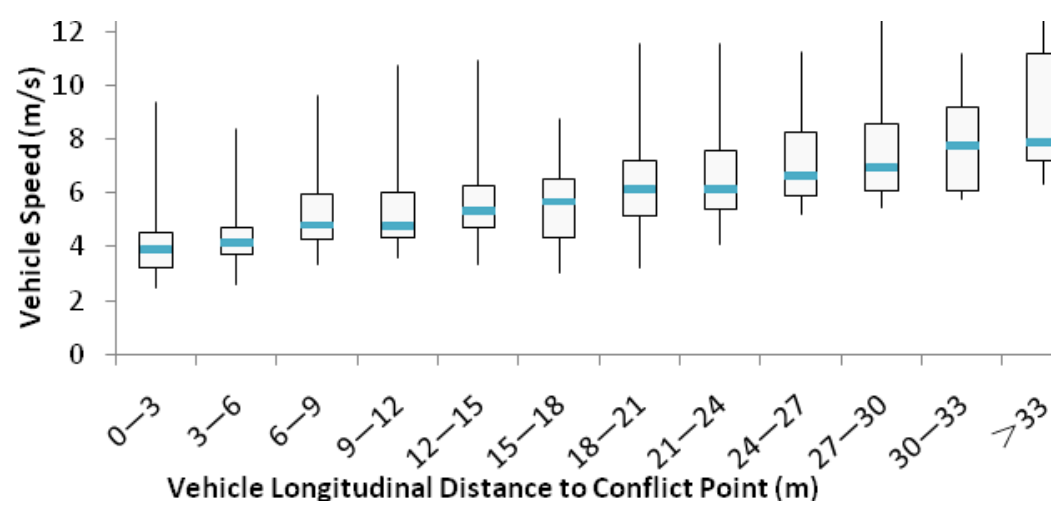

Fig.3 Box plot of relationship between vehicle speed and its longitudinal distance to conflict point in Situation I

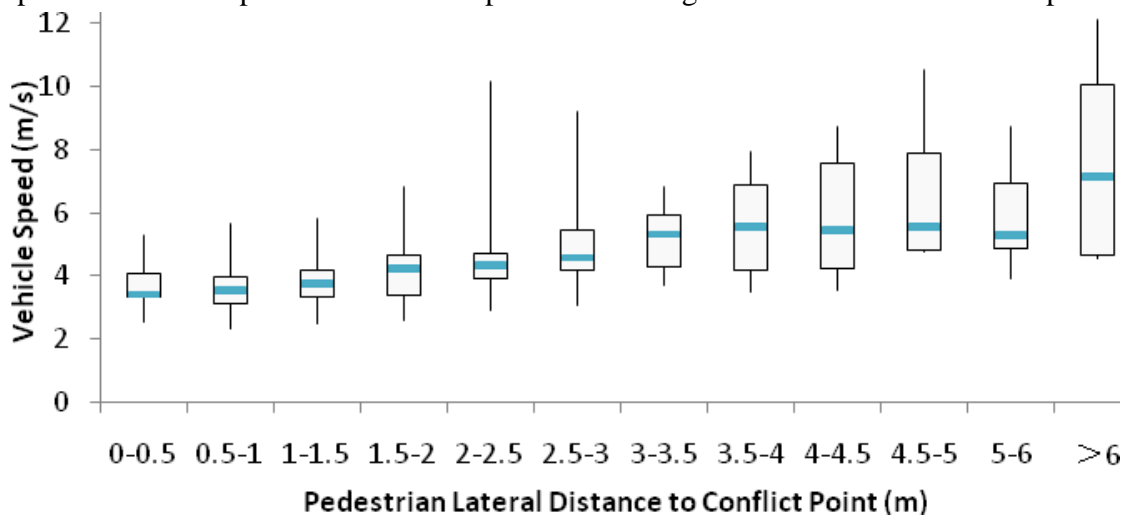

Fig.4 Box plot of relationship between vehicle speed and pedestrian lateral distance to conflict point in Situation I 
the jaywalking pedestrians. Fig.4 gives the relationship between vehicle speed and pedestrian lateral distance to conflict point in Situation I . It shows obviously that the pedestrian lateral distance to the conflict point affects the drivers speed choice in yielding behavior. Pedestrian speed will also have an effect on the drivers' decision to a deceleration rate. In previous studies ${ }^{18}$, pedestrian speed could be conducted as input of the perceptive-cognitive driver systems in which the deceleration was the output and a pedestrian speed at $1.5 \mathrm{~m} / \mathrm{s}$ was elicited to be sensitive to the driver.

\subsection{Vehicle in Platoon Encounter with Jaywalking Pedestrian}

The interrelationship process between the jaywalking pedestrian and the vehicle in platoon is very complicated. The violation process of the pedestrian can be divided into several steps

- $\quad$ Arriving at the road side and attempts to violate;
- $\quad$ Forced to wait at the roadside curb;

- $\quad$ Entering the crosswalk and heads toward the road;

- Forced to stop by the vehicles and wait on the crosswalk;

- Crossing the road until a driver is willing to give precedence.

To a driver in the vehicle platoon, s/he has to face a combination influence by both the vehicle in front and the jaywalking pedestrian. 63 samples in Situation II are observed including 50 sample drivers in vehicle platoon don't give way to the jaywalking pedestrians and the other 13 drivers do; the yielding percentage is 20.6\%. Compared with the speed performance in Situation I, the vehicle speed in this situation related to the longitudinal distance to conflict point and the pedestrian lateral distance to conflict point is relatively irregular. Fig.5 and Fig.6 illustrate these two relationships respectively.

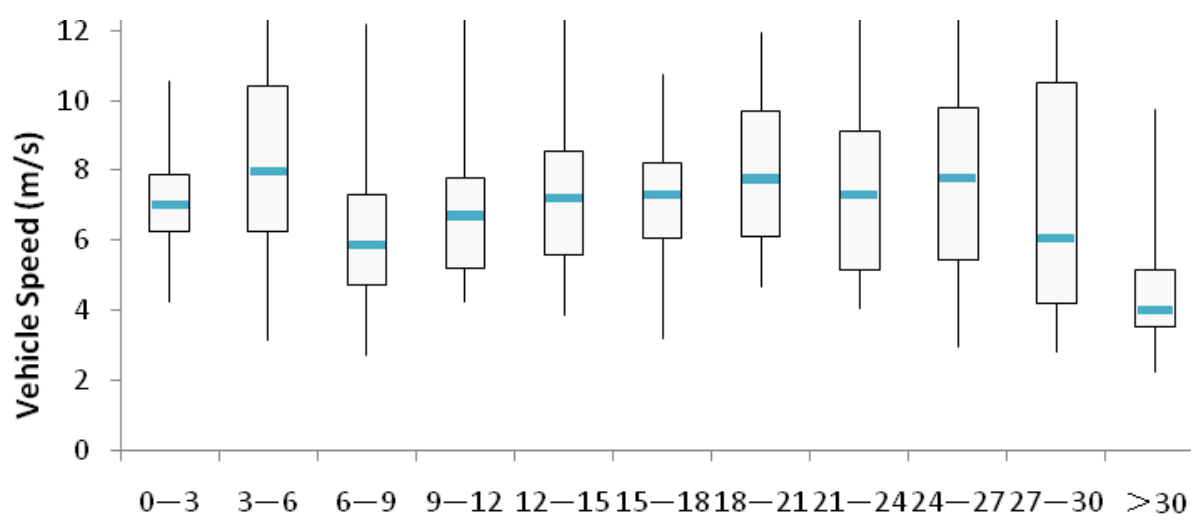

Vehicle Longitudinal Distance to Conflict Point $(\mathrm{m})$

Fig.5 Box plot of relationship between vehicle speed and pedestrian lateral distance to conflict point in Situation II

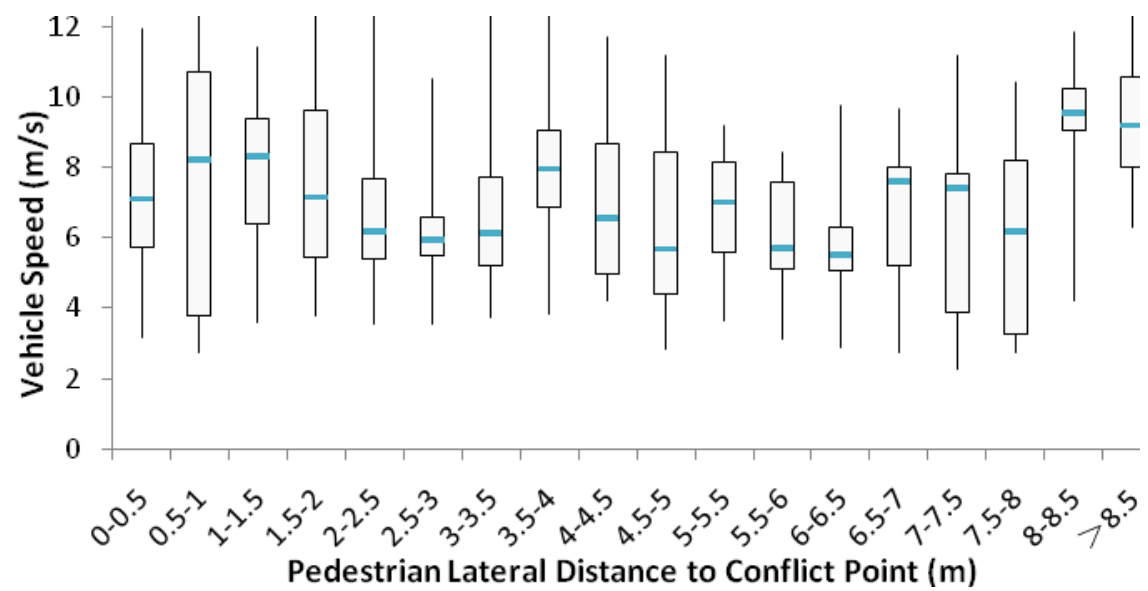

Fig.6 Box plot of relationship between vehicle speed and longitudinal distance to conflict point in Situation II 
Normally, in the car-following phase, the driver in platoon will be influenced by the vehicle in front continuously until the rear bumper of the front vehicle reaches the conflict point on the crosswalk, then the major conflict turns to the conflict between the subject vehicle and the pedestrian. Some parameters characterized this situation have been measured.

- Gap in Distance is defined as when the rear bumper of the vehicle in front reaches the conflict point (complete passing the conflict point), the distance from the rear bumper of the front vehicle to the rear bumper of the subject vehicle (in meter).

- Gap in Time expressed as the time it will take for the subject vehicle to cover the Gap in Distance (in seconds).

Tab.1 gives a statistically description of these parameters assorted by "accept" (the drivers give precedence to the jaywalking pedestrian) and "reject" (the drivers don't give precedence to the jaywalking pedestrian). It can be deduced that if the Gap in Time is larger than $3.5 \mathrm{~s}$, the drivers will prefer to give precedence. Other parameters as the pedestrian speed were calculated according to the trajectories.

\section{Situational Factors Influencing Driver Behavior Based on Logit Model}

It is necessary to the human operator controls the hazardous process work with interpretation of the situation and decision making ${ }^{19}$. For analyzing drivers' giving precedence to jaywalking pedestrian, the flow chart in Fig. 7 illustrates the situational factors as inputs for the driver decision to give precedence during platoon driving.
Firstly when a jaywalking pedestrian presents on the crosswalk, the driver in platoon should determine his/her position in the platoon, and if the vehicle in front is just safely passing the pedestrian that means the subject driver encounters the pedestrian directly. In this case, the situational factors have to been detected, characterized and processed for decision-making. The final decision made by the driver can only be the binary choice: giving precedence or not. The binary logit model which has two discrete choices (generally 1 and 0 ) as its dependent variable can be applied in the study. The probability of choosing either choice is based on a utility function:

$$
U_{t}=\beta_{\alpha}+\beta_{t 1} x_{1}+\beta_{t \Omega} x_{\Sigma}+\cdots+\beta_{t n} x_{n}
$$

Where, $U_{i}$ is the utility of choosing alternative $i$; $\beta_{\text {in }}$ is the coefficient; $i$ is the number of alternatives, and $n$ is the number of the independent variables.

Then the model in terms of odds can be written as:

$$
P(i=1) f(1-F(i=1))=e^{4}
$$

Or in term of the probability of the outcome:

$$
P(l=1)=\frac{1}{1+e^{-z_{l}}}
$$

In this study, five situational factors as model attributes have been conducted in the binary logit model for predicting the drivers' yielding behavior:

- Vehicle Speed (VS): the speed of the subject vehicle, $\mathrm{m} / \mathrm{s}$;

- Lateral Distance of the Jaywalking Pedestrian to the Conflict Point $(L D P)$, m;

- $\quad$ Pedestrian Jaywalking Speed (PS), m/s;

- Position of the Subject Vehicle in Platoon (PSV):

\begin{tabular}{|c|c|c|c|c|c|}
\hline & & \multicolumn{2}{|c|}{ Gap in Time(s) } & \multicolumn{2}{|c|}{ Gap in Distance(m) } \\
\hline & & Reject & Accept & Reject & Accept \\
\hline \multicolumn{2}{|l|}{ Mean } & 2.25 & 4.1 & 18.56 & 26.45 \\
\hline \multicolumn{2}{|c|}{ Std. Error of Mean } & 0.11 & 0.26 & 1.06 & 2.63 \\
\hline \multicolumn{2}{|l|}{ Median } & 2.14 & 3.52 & 16.84 & 24.36 \\
\hline \multicolumn{2}{|c|}{ Std. Deviation } & 0.75 & 0.93 & 0.52 & 9.49 \\
\hline \multirow[t]{4}{*}{ Percentiles } & 5 & 1.21 & 3 & 8.15 & 14.61 \\
\hline & 25 & 1.78 & 3.34 & 11.97 & 19.1 \\
\hline & 75 & 2.6 & 5.16 & 24.03 & 33.93 \\
\hline & 95 & 3.68 & 5.24 & 34.04 & 48.53 \\
\hline
\end{tabular}
when the pedestrian jaywalks from the roadside

Tab.1 Statistics of Gap in Time and Gap in Distance 
curb, the vehicle which is the first to encounter the pedestrian is considered as 1st Position;

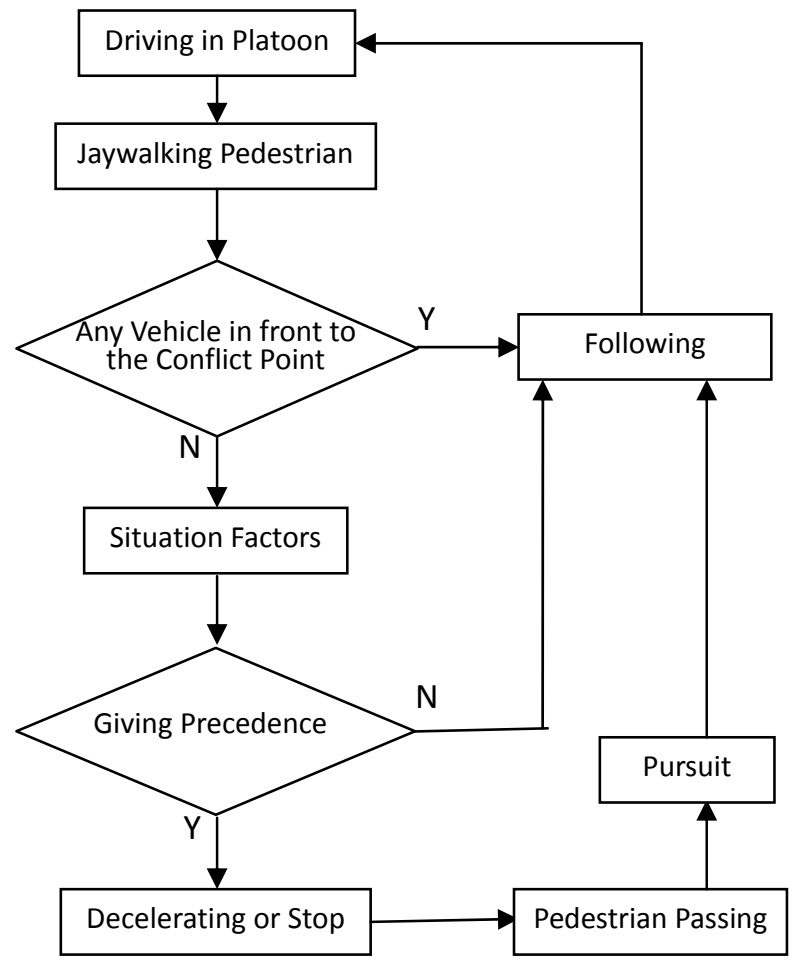

Fig.7 Flow Chart of Platoon Driving

- Gap in Distance (GD): compared with Gap in Time, the distance-centered parameter Gap in Distance can be intuitively perceived by the driver and these two parameters have a linear relationship to a certain extent, so the Gap in Distance is determined as the independent variable in the model, $\mathrm{m}$.

The discrete choice set $(1,0)$ is defined as the output of the model, 1 for the decision of giving precedence to the jaywalking pedestrian and 0 for not giving precedence. The utility function for this model is:

$$
\begin{aligned}
& U_{1}=\beta_{0}+\beta_{1} \cdot V S+\beta_{2} \cdot L D P+\beta_{8} \cdot P S+\beta_{4} . \\
& P S V+\beta_{Q} \cdot G D
\end{aligned}
$$

After logistic regression and variable test, the four independent variables VS, LDP, $P S$ and $G D$ are significant $(\mathrm{p}<0.05)$, so we remove the non-significant situational factor PSV from the model. The probability of the drivers' giving precedent can be predicted by:

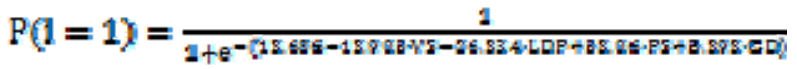

55 sample data series were used for estimating the coefficients of the model by regression and the other 8 samples for prediction. The result showed that the binary logit model could predict the drivers' yielding behavior accurately (the predicted value were all fit the observed data). The model also specified the vehicle speed, the lateral distance of the jaywalking pedestrian to the conflict point, the pedestrian speed and the gap in distance as the situational factors had an influence to the driver behavior in giving precedence to jaywalking pedestrian while the vehicle position in the platoon didn’t significantly affect such behavior.

\section{Concluding remarks and future works}

The driver behavior centered encounters with jaywalking pedestrians at signalized crosswalk has been observed and measured in this paper. By analysis on the trajectories of both the vehicles and the jaywalking pedestrians, the characteristics of road user behavior can be derived. Drivers' speed performance has been studied related to the lateral distance of the jaywalking pedestrian to the conflict point and the vehicle longitudinal distance to the conflict point. The speed performance between Situation I and Situation II are significantly different due to the complexity of the traffic conditions. The box plots implied a linear decrease of the vehicle speed in approaching process in the former situation versus irregular way in the latter one. Two definitions, Gap in Distance and Gap in Time have been introduced and statistically estimated for drivers' decision of giving precedence. In this study, binary logit model is applied to estimate the drivers' yielding behavior influenced by the situational factors. Four factors: $V S, L D P, P S$ and $G D$ are demonstrated to have great effect to the driver decision in giving precedence in platoon driving and the probability function can predict the drivers' choices accurately. However, lack of detection and taxonomies to the individual characters of pedestrians like the gender, age, assertiveness, disability, etc., and the feature parameters of the roadway, the situational factors proposed here cannot describe the entire conflict condition comprehensively. More 
situational information needs to be gathered in the further work and the model proposed in this study could hopefully be the groundwork in the target of modeling driver behavior in vehicle-pedestrian conflicts.

\section{Acknowledgements}

This work was supported in part by Alexander von Humboldt Foundation under grant 1072467 and the National Natural Science Foundation of China under grant 50878023.

\section{References:}

1. R. Retting, F. S. Ferguson, and A. McCartt, Anne, A review of evidence-based traffic engineering measures designed to reduce pedestrian-motor vehicle crashes, American Journal of Public Health, 93(9) (2003) 1456-1463

2. German Federal Statistical Office, Persons involved in and casualties from accidents

(http://www.destatis.de/jetspeed/portal/cms/Sites/destatis /Internet/EN/Content/Statistics/Verkehr/Verkehrsunfaelle /Tabellen/Content50/PersonsKilledTrafficParticipation,te mplateId=renderPrint.psml, 2010).

3. Ministry of Public Security of People's Republic of China (MPSPRC). Annual Report of the China Road Traffic Accidents Statistics. (Beijing, China, 2003, 2004, 2007).

4. J. Qiu, Analysis on causes, features and trend of road traffic crashes and traffic injuries in China. (Doctoral thesis, Medical Colleges of PLA, China, 2009)

5. E. Pasanen, Driving speeds and pedestrian safety; a mathematical model, (Helsinki University of Technology, Transportation Engineering, Publication 77, Otaniemi, Finland, 1992).

6. U. Engel, L. K. Thomsen, EVekter af fardselslovens '40-Sammenfatning. (Rapport 29, Ra ${ }^{\circ} \mathrm{det}$ for Trafiksikkerhedsforskning, København, Denmark, 1990).

7. E. J. Westra, J. A. Rothengatter, Behaviour -Conflict -Safety Relations for Pedestrians, (Traffic Research Centre, University of Groningen, Netherlands, 1993).

8. S. Johannessen, Road User Behaviour, Safety and Design of Non-Signalised Pedestrian Crossings. A Challenging Interaction Dilemma, in Proc. of 9th International Conference for Walking (Barcelona, Spain 2008).

9. K. Aronsson, Speed characteristics of urban streets based on driver behavior studies and simulation, (Doctoral thesis, Stockholm, Sweden, 2006).

10. A. Varhelyi, Driver Speed Behavior at a Zebra Crossing: A Case Study, Accident Analysis and Prevention, 30(6) (1998) 731-743.
11. H. Persson, Communication between Pedestrian and Car Drivers, (Lund University, Lund, Sweden, 1988).

12. A. Katz, D. Zaidel and A. Elgrishi, An experimental study of driver and pedestrian intersection during the crossing conflict, Hum Factors, 17 (1975) 514-527.

13. V. Himanen, and R. Kulmala, An application of logit models in analyzing the behavior of pedestrians and car Drivers on pedestrian crossings. Accident Analysis and Prevention, 20(3) (1988) 187-197.

14. D. Geruschat and S. Hassan, Driver behavior in yielding to sighted and blind pedestrians at roundabouts. Journal of Visual Impairment \& Blindness, 99 (2005) 286-302.

15. D. Sun, S. Ukkusuri, R. Benekohal and S. Travis, Modeling of motorist-Pedestrian interaction at uncontrolled mid-block crosswalks. in Proc. of TRB 2003 Annual Meeting (Washington, DC, USA,2003).

16. B.J. Schroeder, A Behavior-based methodology for evaluating pedestrian-vehicle interaction at crosswalk, $(\mathrm{PhD}$ Dissertation, North Carolina State University, United States, 2008).

17. C. Nowakowski, Pedestrian Warning Human Factor Considerations. Retrieved

(http://path.berkeley.edu/_cychan/Research_and_Present ation/Pedestrian_Detection_TO5200/Crosswalk_HF.pdf. 2005)

18. X. Jiang, W. Wang, K. Bengler, W. Guo, Driver behavior centred traffic conflict with pedestrian at signalized intersection crosswalk. Journal of Beijing Institute of Technology (English Edition), 19(2) (2010) 16-20.

19. Wang. W., Zhang, W., Guo, H., Bubb, H., Ikeuchi, K., A safety-based behavioral approaching model with various driving characteristics, Transportation Research Part C, doi:10.1016/j.trc.2011.02.002

20. Wang W., Guo H., Gao Z., Bubb H., Individual differences of pedestrian behaviour in midblock crosswalk and intersection, International Journal of Crashworthiness, 16(1), 1-9

21. Kelly J. Clifton, Carolina V. Burnier, Gulsah Akar, Severity of injury resulting from pedestrian-vehicle crashes: What can we learn from examining the built environment? Transportation Research Part D, 14(6), 2009, 425-436

22. A. Shalom Hakkert, Victoria Gitelman, Eliah Ben-Shabat, An evaluation of crosswalk warning systems: effects on pedestrian and vehicle behavior. Transportation Research Part F ,5 (2002) 275-292.

23. Wang, W. Jiang, X., Xia, S. Cao, Q., Incident tree model and incident tree analysis method for quantified risk assessment: an in-depth accident study in traffic operation, Safety Science, 48(10) (2010),1248-1262

24. Sytze A. Rienstra, Piet Rietveld, Speed behaviour of car drivers: A statistical analysis of acceptance of changes in speed policies in the Netherlands, Transportation Research Part D,1(2), 1996, 97-110. 